with bleomycin that is known to induce fibrotic disease in otherwise normal $\mathrm{C} 57 / \mathrm{Bl} 6$ mice, and monitored the animals for disease phenotype.

Methods We injected MRL-MpJ-Fas lpr/lpr (MRL-lpr) mice with bleomycin $(100 \mu \mathrm{g}$, subcutaneously on upper back) or PBS daily for 14 days. Following which, animals were monitored daily for 14 days. Photographs were taken for skin lesions. On day 28, skin, paws, lung, liver, and kidneys were harvested and tissues sectioned for $\mathrm{H}$ and $\mathrm{E}$ and Masson Trichrome staining. Immunohistochemistry was performed to detect blood vessels and endothelial cells, namely -smooth muscle actin (SMA) and CD31, respectively. Experiments were repeated in MRL-MpJ-Fas $+/+(\mathrm{MRL}+/+)$ mice.

Results Around day 10 of injections, the tips of front and hind paw digits of 7 of 10 bleomycin-injected MRL-lpr and 3 of 3 bleomycin-injected MRL+/+mice developed erythematous lesions that ulcerated. None of the controls (5 PBS-injected MRL-lpr, 3 PBS-injected MRL+/+, and 3 bleomycin-injected C57/Bl6 mice) developed such lesions. Such lesions have also not been observed in over 50 unmanipulated MRL-lpr and $\mathrm{MRL}+/+$ mice or over 30 bleomycin-injected C57/Bl6 mice in our animal colony in previous studies. Histological and immunohistochemistry analyses showed increased infiltration, fibrosis, tissue destruction, and CD31 expression in bleomycininjected MRL-lpr mice as compared to control animals. Massons trichrome staining revealed significantly increased dermal fibrosis in bleomycin-injected MRL-lpr mice as compared to PBS-injected MRL-lpr mice. Preliminary analysis shows increased alveolar hemorrhage in bleomycin-injected MRL-lpr mice as compared to control mice.

Conclusions The vasculitic lesions that we observed in the digits of bleomycin-injected MRL-lpr and MRL $+/+$ mice mimics vasculitic lesions seen in patients with lupus. Thus, bleomycin injection in lupus-prone mice can serve as a model for chronic vascular changes seen in lupus and other systemic rheumatic diseases.

Funding Source(s): None

\section{EPIGENOME-WIDE ASSOCIATION STUDY REVEALS DIFFERENTIAL DNA METHYLATION IN SYSTEMIC LUPUS ERYTHEMATOSUS PATIENTS WITH A HISTORY OF ISCHEMIC HEART DISEASE}

${ }^{1}$ Juliana Imgenberg-Kreuz* ${ }^{2}$ Christopher Sjöwall, ${ }^{2}$ Martina Frodlund, ${ }^{3}$ Iva Gunnarsson, ${ }^{3}$ Elisabet Svenungsson, ${ }^{1}$ Dag Leonard. ${ }^{1}$ Uppsala University; ${ }^{2}$ Linköping University; ${ }^{3}$ Karolinska Insitutet

10.1136/lupus-2019-Ism.12

Background Patients with systemic lupus erythematosus (SLE) have an increased risk of cardiovascular disease (CVD), including ischemic heart disease (IHD). We performed a case-case epigenome-wide association study (EWAS) for IHD in patients with SLE to identify phenotype-specific differences in DNA methylation.

Methods DNA methylation in peripheral blood samples from two independent cohorts of Swedish SLE patients $(n=347$ and $\mathrm{n}=201$, respectively) was assayed on the HumanMethylation450k BeadChip array, targeting 485,000 CpG sites across the genome. Clinical data were retrieved from medical charts and individuals with a history of CVD were identified in both cohorts. Differential DNA methylation between SLE patients with a history of IHD ( $n=20$ and $n=17$, respectively) and SLE patients without any CVD events prior to DNA sampling was tested using a logistic regression model including age, sex and cell type distribution as covariates. Differentially methylated $\mathrm{CpG}$ sites in the discovery cohort were defined as $\mathrm{p}<1.3 \mathrm{E}-7$ for association based on Bonferroni correction and an absolute average difference in methylation beta of $\|$ $>0.05$. Significance in the replication cohort was determined as $\mathrm{p}<0.05$ and same direction of effect.

Results The top associated differentially methylated CpG sites that were replicated were identified at programmed cell death 1 (PDCD1, p (disc) $=3.2 \mathrm{E}-13 ; \mathrm{p}(\mathrm{repl})=0.03)$, perforin 1 (PRF1, $\mathrm{p}($ disc $)=1 \mathrm{E}-12 ; \mathrm{p}(\mathrm{repl})=0.03)$ and $\mathrm{ZFP} 36$ ring finger protein like 1 (ZFP36L1, $p$ (disc)=1.3E-11; $\mathrm{p}(\mathrm{repl})=0.002)$, all of which are implicated in apoptotic processes. Functional pathway analysis of genes containing sites with altered methylation in SLE IHD pointed to muscle contraction $(p=4.3 \mathrm{E}-10)$, cardiac conduction $(p=2.2 \mathrm{E}-7)$ and role of agrin in postsynaptic differentiation $(p=2.9 \mathrm{E}-7)$ as the most significantly enriched pathways.

Conclusions The results of this study highlight genes and pathways that may be implicated in the pathogenesis of and/or recovery from IHD in patients with SLE. The differentially methylated $\mathrm{CpG}$ sites identified in this study can serve as candidates for further evaluation by functional studies and as potential biomarkers for IHD in patients with SLE.

Funding Source(s): None

\section{THE ASSOCIATION BETWEEN THE DOSE OF GLUCOCORTICOIDS AND HEALTH-RELATED QUALITY OF LIFE IN PATIENTS WITH SYSTEMIC LUPUS ERYTHEMATOSUS: A CROSS-SECTIONAL STUDY}

${ }^{1}$ Yoshia Miyawaki, ${ }^{1}$ Sayaka Shimizu* ${ }^{1}$ Yusuke Ogawa, ${ }^{2}$ Ken-ei Sada, ${ }^{3}$ Kunihiro Ichinose, ${ }^{4}$ Ryusuke Yoshimi, ${ }^{5}$ Shigeru Ohno, ${ }^{6}$ Nobuyuki Yajima, ${ }^{1}$ Shunichi Fukuhara. ${ }^{1}$ Department of Healthcare Epidemiology, School of Public Health in the Graduate School of Medicine, Kyoto University; ${ }^{2}$ Department of Nephrology, Rheumatology, Endocrinology and Metabolism, Okayama University Graduate School of Medicine, Dentistry and Pharmaceutical Sciences; ${ }^{3}$ Department of Immunology and Rheumatology, Advanced Preventive Medical Sciences, Graduate School of Biomedical Sciences, Nagasaki University; ${ }^{4}$ Department of Stem Cell and Immune Regulation, Yokohama City University Graduate School of Medicine; ${ }^{5}$ Center for Rheumatic Diseases, Yokohama City University Medical Center; ${ }^{6}$ Division of Rheumatology, Department of Internal Medicine, Showa University School of Medicine

\subsection{6/lupus-2019-Ism.13}

Background Glucocorticoids (GC) is a mainstay of treatment for systemic lupus erythematosus (SLE) but generally known to affect the health-related quality of life (HRQOL). The aim in this study is to investigate the association between the current dose of GC and HRQOL in SLE patients.

Methods This was a cross-sectional study using baseline data of nationwide SLE patients registry (LUNA) from April 2018 through September 2018. Participants were outpatients with SLE aged 20 years or older. The exposure was the current dose of GC (an equivalent of daily prednisolone). The primary outcome was the HRQOL score of Lupus Patient Reported Outcome (LupusPRO). We included age, sex, and damage in covariates to be particularly considered for the effect of GC-related damage. Damage was divided into GCrelated damage and other damage using the SLICC damage index (SDI). GC-related damage was defined as the presence of diabetes mellitus, osteonecrosis, osteoporotic fractures and cataracts. We used a linear regression model to assess the association between the current dose of GC and the HRQOL and further evaluated which of the current daily dose of GC 
Abstract 13 Table 1 Multiple linear regression analysis for the HRQOL

\begin{tabular}{|c|c|c|c|c|}
\hline Variables & $\begin{array}{c}\text { Co- } \\
\text { efficient }\end{array}$ & $\begin{array}{l}95 \% \mathrm{Cl} \\
\text { Lower }\end{array}$ & $\begin{array}{l}95 \% \mathrm{Cl} \\
\text { Upper }\end{array}$ & $\begin{array}{c}\text { Standardized } \\
\beta\end{array}$ \\
\hline Intercept & 92.52 & & & \\
\hline (mg daily) & -0.88 & -1.45 & -0.31 & -0.23 \\
\hline (years old) & -0.07 & -0.29 & 0.15 & -0.05 \\
\hline male [reference] & -13.62 & -21.35 & -5.90 & -0.25 \\
\hline $\begin{array}{l}\text { GC-related SDI >1 } \\
\qquad \mathrm{SDI}=0 \text { [reference] }\end{array}$ & -8.14 & -14.56 & -1.72 & -0.20 \\
\hline $\begin{array}{l}\text { GC-unrelated SDI >1 } \\
\text { SDI=0 [reference] }\end{array}$ & -5.04 & -10.92 & 0.83 & -0.12 \\
\hline
\end{tabular}

HRQOL; Health-Related Quality of Life, SLEDAl; Systemic Lupus Erythematosus Disease Activity Index, 95\% Cl; 95\% confidence interval, GC; Glucocorticoid (prednisolone-equivalent dose), SDI; Systemic Lupus International Collaborating Clinics/American College of Rheumatology Damage Index.

and the GC-related damage has a greater impact on HRQOL from standardized -coefficients. Multiple imputation was performed for missing data.

Results Of the 188 enrolled patients, 84\% were female and the median age was 44 (interquartile range [IQR] 35-55) years. The median SLEDAI was 4 (IQR 2-8) and the median of daily prednisolone dosage was 5 (IQR 4-9) mg. The median HRQOL score was 70 (IQR 53-84). HRQOL was significantly associated with the daily dose of prednisolone $(=-0.50 \quad[95 \%$ confidence interval $(\mathrm{CI})-0.99$ to -0.02$])$, SDI $1(=-8.2[95 \% \mathrm{CI}-14.0$ to -2.4$])$ and female $(=-11.8$ [95\%CI -19.8 to -3.9$])$. Multiple linear regression analysis showed that the daily prednisolone dose was significantly associated with HRQOL $(=-0.88$ [95\%CI -1.45 to -0.31$])$. The current daily dose of GC had a greater influence on HRQOL than the GC-related damage (standardized $=-0.23$ vs standardized $=-0.20$ ). After multiple imputation of missing values, our findings did not substantially change $(=-0.66$ [95\%CI -1.14 to -0.17$])$.

Conclusions The daily dose of GC was associated with HRQOL among SLE patients rather than the GC-related damage. These findings may help to understand the effects of GC treatment on HRQOL.

Funding Source(s): None

\section{NOT HAVING THE REAL SUPPORT THAT WE NEED: PATIENTS EXPERIENCES WITH AMBIGUITY OF SYSTEMIC LUPUS ERYTHEMATOSUS AND EROSION OF SOCIAL SUPPORT}

${ }^{1}$ Jerik Leung ${ }^{*},{ }^{2}$ Elizabeth Baker, ${ }^{1}$ Alfred H Kim. ' Washington University School of Medicine; ${ }^{2}$ Saint Louis University College for Public Health and Social Justice

10.1136/lupus-2019-Ism.14

Background A hallmark of SLE is the paroxysmal disease course with symptoms fluctuating unpredictably both across different individuals and within individual cases. Importantly, the patient-specific experience of living with SLE is underreported, particularly when studying factors associated with health-related quality of life (HRQOL). Recent work has suggested that biomedical interventions are only partially predictive of HRQOL measures. The patient specific experience studied using qualitative methods is a necessary initial step to uncover additional root causes of poor HRQOL in SLE populations as patient experiences add richness and depth to a topic of inquiry not possible through quantitative methods.

Methods Consented adult patients with American College of Rheumatology- or Systemic Lupus International Collaborating Clinics-classified SLE were recruited for this study during their scheduled clinic visits. Ten semi-structured interviews were conducted across six participants. Interviews were audio recorded, transcribed, and analyzed using an iterative process where all data were first grouped based on the major questions of the study and then coded using an open coding scheme, allowing for identification of key issues based on patient experiences. Findings were presented to an interactive public forum with SLE patients, family members and friends of individuals with SLE, and health care professionals and assessed for accuracy and credibility.

Results Four major factors that influence HRQOL emerged from the interviews: 1) ambiguity, inconsistency, and lack of symptom predictability due to SLE disease courses, 2) poor communication with family/friends/partners, and poor bi-directional communication between health care providers and patients (informational support), 3) lack of validation for patients experiences (appraisal support), and 4) problematic aspects of social support including negative support and patients inability to reciprocate support due to role changes. Data also indicate a reciprocal association between appraisal and informational sources of support.

Conclusions Findings indicate that inadequate appraisal and informational support from informal and formal sources and ambiguity are particularly salient factors influencing HRQOL 